\title{
Identifying the patterns of changes in $\alpha$ and $\beta$ diversity across Dacrydium pectinatum communities in Hainan Island, China
}

\author{
Haodong Liu ${ }^{1}$, Hua Liu ${ }^{1}$, Yongfu Chen ${ }^{1}$, Zhiyang $\mathrm{Xu}^{2}$, Yunchuan Dai ${ }^{1}$, and Qiao Chen ${ }^{1}$ \\ ${ }^{1}$ Chinese Academy of Forestry \\ ${ }^{2}$ Affiliation not available
}

October 9, 2020

\begin{abstract}
Exploring vegetation distribution spatial patterns facilitates understanding how biodiversity addresses the potential threat of future climate variability, especially for highly diverse and threatened tropical plant communities, but few empirical studies have been performed over various environmental scales. In this study, we used species-based and phylogeny-based methods to analyze the $\alpha$ - and $\beta$-diversity pattern variation in Dacrydium pectinatum communities and its key drivers along elevation and geographical gradients across three national nature reserves in Bawangling, Diaoluoshan and Jianfengling. Our study indicates that the species and phylogenetic $\alpha$-diversity patterns presented consistent decreasing with elevation, with the peak occurring at low elevations. Environmental filtering caused by decreases in limiting factors, such as temperature, precipitation, soil organic matter, soil phosphorus, and light, is the main reason for the decline in diversity at high elevations, whereas low-elevation areas are affected by various factors, such as environmental filtering and similarity limitations. Species and phylogenetic $\beta$-diversity changes are closely related to environmental filtering and dispersal limitation, but the latter is key in community assembly at the heterogeneous spatial scale. In conclusion, combining species-based and phylogeny-based methods to explore the biogeographic patterns of tropical plant communities helps provide convincing evidence and confirms that the relative contributions of niche and neutral theory in the assembly process vary along environmental gradients. Though the D. pectinatum community constitute a floristically integrated unit, the genetic relationships between species are relatively far, and co-evolution to promote species coexistence is difficult when faced with habitat pressure. Hence, we believe that species coexistence in tropical plant communities requires mild environmental conditions, and low temperatures, precipitation, soil nutrients and light will aggravate environmental filtering and species competition. We also recommend strengthening the construction and management of nature reserves and the exploration of biodiversity formation mechanisms, which are crucial for biodiversity conservation in endangered tropical plant communities.
\end{abstract}

\section{Hosted file}

Manuscript.pdf available at https://authorea.com/users/317678/articles/485677-identifyingthe-patterns-of-changes-in-\%CE\%B1-and-\%CE\%B2-diversity-across-dacrydium-pectinatumcommunities-in-hainan-island-china 


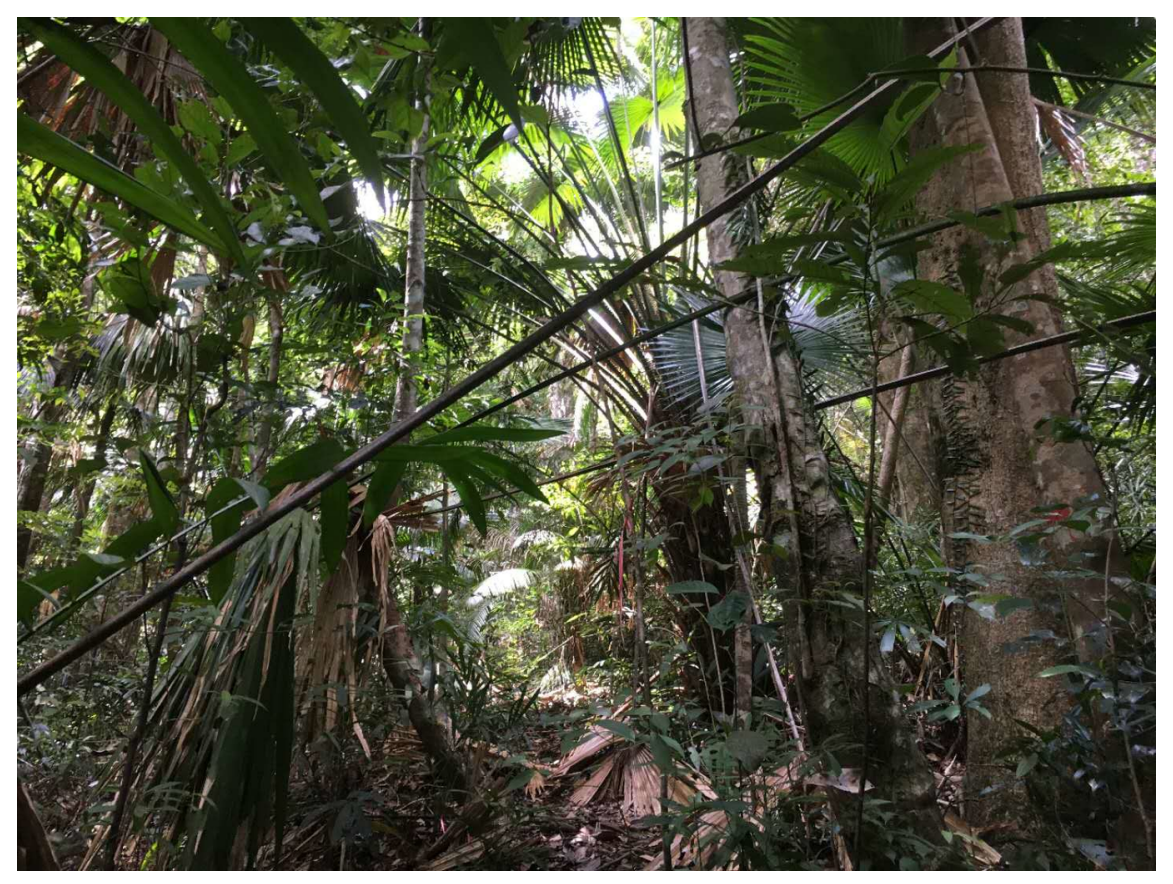

